\title{
Simple Connectivity in Polar Spaces
}

\author{
Max Horn Reed Nessler Hendrik Van Maldeghem
}

\author{
Mathematisches Institut \\ Justus-Liebig-Universität Gießen \\ Arndtstrasse 2, \\ D-35392 Gießen \\ max.horn@math.uni-giessen.de
}

\author{
Department of Mathematics \\ University of Virginia \\ 401 Kerchof Hall, \\ Virginia VA 22904-4137, USA \\ rcn9f@virginia.edu
}

\author{
Department of Mathematics \\ Ghent University \\ Krijgslaan 281-S22, \\ B-9000 Ghent \\ hvm@cage. ugent. be
}

\begin{abstract}
We settle the simple connectivity of the geometry opposite a chamber in a polar space of rank 3 by completing the job for the non-embeddable polar spaces, and some polar spaces with small parameters.
\end{abstract}

Keywords: Non-embeddable polar space, opposition, covering degree, simple connectivity

MSC: $51 \mathrm{E} 24$

\section{Introduction}

In 1996, Peter Abramenko [1] showed that the geometry $\Delta^{0}(C)$ induced by the set of chambers opposite a given chamber $C$ in "most" rank 3 polar spaces $\Delta$ is simply connected. Besides some polar spaces with small parameters, the main rather annoying open case was the family of so-called non-embeddable polar spaces. Due to lack of an efficient, explicit and suitable description, this case remained open ever since. In this paper, we will prove that $\Delta^{0}(C)$ is simply connected, for any chamber $C$ of a non-embeddable polar space $\Delta$, using the recent coordinate description by De Bruyn \& Van Maldeghem [4]. Also, we settle the cases of classical polar spaces with small parameters, which are not covered by Abramenko's result, and we determine the covering degree. For the small cases, we use computer computations.

The simple connectivity of $\Delta^{0}(C)$ is used in a number of different situations and results. All these results excluded, up to now, the non-embeddable polar spaces and some small polar spaces of rank 3. As a corollary to Theorems 2.1 and 2.2 below, all those results now also hold for the non-embeddable polar spaces, and some small classical polar spaces. Let us mention three examples.

1. Abramenko's original motivation. From the simple connectivity of $\Delta^{0}(C)$ for a rank 3 building $\Delta$ and a chamber $C$ in $\Delta$, or, more generally, from the $(n-1)$ sphericity of $\Delta^{0}(C)$ if $\Delta$ has rank $n$, Abramenko deduces in [1] finiteness properties 
of certain $S$-arithmetic groups related to locally finite function fields, say with local field $\mathbb{F}_{q}$. The results in [1] now also hold for some smaller values of $q$ than mentioned there.

2. Group amalgamations. In [14, Tits wants to determine when the unipotent subgroup of a group with a (split) spherical BN-pair is the amalgamated sum of its "rank two subsystems" (the subgroups generated by two root groups in $U$ ). This is precisely the case when $\Delta^{0}(C)$ is simply connected, where $\Delta$ is the corresponding (Moufang) building, and $C$ is the chamber fixed by $U$, see Proposition 6 of [14]. Our results imply that this condition is satisfied for the building corresponding to the thick non-embeddable polar spaces. Similarly, but using different methods, Devillers \& Mühlherr [5] prove that, the (positive or negative) Borel subgroup of every Kac-Moody group defined over a finite field with at least 16 elements is finitely presented. This bound can now be sharpened to "at least 5 elements".

3. A rank 3 criterion for extending a twinning to a twin building. In [6], Devillers, Mühlherr \& Van Maldeghem prove a rank 3 criterion for a twinning of a chamber with a (2-spherical) building $\Delta$ to extend to a full twin building. This criterion contains the condition that for all spherical rank 3 residues $\Delta$ and every chamber in $\Delta$, the geometry $\Delta^{0}(C)$ is simply connected. Our results imply that this is fine for rank 3 residues isomorphic to buildings corresponding to non-embeddable polar spaces, and for some small polar spaces of rank 3. More precisely, the conditions mentioned in Corollary 1 of [6] saying that "the planes of the polar space must be Desarguesian and every panel must be contained in at least 17 chambers" can be changed to "every panel must be contained in at least 6 chambers".

\section{Main Results}

A polar space $\Delta$ of rank $n+1, n \geq 1$, is a geometry of rank $n+1$ with type set $\{0,1, \ldots, n\}$ (this is just an $(n+1)$-partite graph where the classes are labeled 0 up to $n$ ) satisfying the following axioms (see [13, Chapter 7] or [15]), where we call the elements of type 0 points. In the following, a projective space of dimension -1 is the empty set, of dimension 0 is a singleton and of dimension 1 is a set of at least 3 points.

(PS1) The elements of type $<i$ incident with any element of type $i$ form naturally a projective space of dimension $i$ in which the type of an element in $\Delta$ is precisely the dimension of the corresponding subspace in that projective space.

(PS2) Every element of $\Delta$ is determined by the set of points incident with it and the point sets of two elements of $\Delta$ intersect in a subspace of each.

(PS3) For every point $x$ and every element $M$ of type $n$ not incident with $x$, there exists a unique element $M^{\prime}$ through $x$ of type $n$ whose point set intersects the point set of $M$ in the point set of an element of type $n-1$. Also, no element of type 1 is incident with $x$ and a point of $M$ unless it is incident with $M^{\prime}$ or coincides with $M^{\prime}$. 
(PS4) There exist two elements of type $n$ not incident with any common point.

A polar space of rank $n+1$ is called thick if every element of type $n-1$ is incident with at least three elements of type $n$; it is called non-thick otherwise. Usually, we view the elements of type $i$ as subsets of the set of points, and we call them singular $i$-spaces. Singular $n$-spaces are maximal singular subspaces. We call two points collinear when they are contained in a common 1-space. A generalized quadrangle is a polar space of rank 2 or the dual of it. A thick generalized quadrangle is a thick polar space of rank 2 and its dual is always again a thick polar space of rank 2 .

The incidence relation of a polar space coincides with the adjacency of the corresponding $(n+1)$-partite graph, which we call the incidence graph of the polar space. We will use both notions. By the geometry induced by a subset $S$ of $\Delta$, we mean the induced subgraph. We will identify $S$ with the geometry it induces and, in particular, talk about the connectivity of $S$ (which means the connectivity of the graph induced by $S$ in the incidence graph). The distance between two elements is the distance measured in the incidence graph. The distance between elements $x$ and $y$ is denoted by $d(x, y)$. For an element $x$ of $\Delta$ we denote by $\Delta_{x}$ the set (or induced subgraph on the set) of neighbors of $x$ in $\Delta$. Note that in case the rank of $\Delta$ is 3 , if $x$ is a point, then $\Delta_{x}$ is itself a generalized quadrangle; if $x$ is a line, then it is a generalized digon (a complete bipartite graph); if $x$ is a plane, then it is a projective plane.

In essence, a polar space is the natural geometry of a pseudo-quadratic form, as introduced by Jacques Tits in [13]. They arise as subgeometries of projective spaces (the points are precisely the points of the projective space where the pseudo-quadratic form vanishes), and are hence called the embeddable polar spaces. Since the corresponding groups are classical groups, one also calls them classical polar spaces. Axiomatizing the situation, in the light of the introduction of Tits-buildings, aiming at a uniform description of all geometries naturally associated with simple algebraic groups, there appear two classes of polar spaces which cannot be described using a pseudo-quadratic form. The first class consists of the line Grassmanian geometries of 3-dimensional projective spaces over non-commutative fields, and these are considered to be the trivial (non-thick) nonembeddable polar spaces (they are also classical in the sense that their automorphism groups are classical linear projective groups). The second class is related to exceptional groups of type $E_{7}$ and the Cayley planes; every such polar space is thick, has rank 3 and its planes are projective planes coordinatized by Cayley-Dickson division algebras. The latter implies that these polar spaces cannot be embedded in any projective space, and hence they are called the non-embeddable polar spaces, or also the non-classical polar spaces.

In the finite case, every polar space arises from a symplectic polarity, or a non-degenerate Hermitian variety, or a non-degenerate quadric. In projective space $\mathrm{PG}(2 n+1, q)$ of odd dimension $2 n+1$ over the Galois field $\mathbb{F}_{q}$, we have the (thick) symplectic polar space $\mathrm{W}(2 n+1, q)$ (arising from a symplectic polarity) of rank $n+1, n \geq 1$, the (thick) Hermitian polar space $\mathrm{H}(2 n+1, q)$ (arising from a non-degenerate Hermitian variety) of rank $n+1, n \geq 1$, the (thick) elliptic polar space $\mathrm{Q}^{-}(2 n+1, q)$ (arising from an elliptic quadric) of rank $n, n \geq 2$, and the (non-thick) hyperbolic polar space $\mathrm{Q}^{+}(2 n+1, q)$ 
(arising from a hyperbolic quadric) of rank $n+1, n \geq 1$. In projective space PG $(2 n, q)$ of even dimension $2 n$ over the Galois field $\mathbb{F}_{q}$, we have the (thick) Hermitian polar space $\mathrm{H}(2 n, q)$ (arising from a non-degenerate Hermitian variety) of rank $n, n \geq 2$, and the (thick) parabolic polar space $\mathrm{Q}(2 n, q)$ (arising from a parabolic quadric) of rank $n+1$, $n \geq 1$. The polar spaces $\mathrm{W}(2 n+1, q)$ and $\mathrm{Q}(2 n+2, q)$ are isomorphic if $q$ is even.

Let $\Delta$ be a polar space of rank $n+1, n \geq 1$. A flag is a set of pairwise incident elements, and a chamber is a flag consisting of $n+1$ elements. Two elements $A, B$ are called opposite if no point of one element is collinear with all points of the other element. Two flags are opposite if every member of one flag is opposite some member of the other flag. Let $C$ be a chamber of $\Delta$. Then $\Delta^{0}(C)$ is the rank $n+1$ geometry induced on the union of all chambers opposite $C$. More generally, if $F$ is a flag of $\Delta$, then $\Delta^{0}(F)$ is the rank $n+1$ geometry induced on the union of all chambers containing a flag opposite $F$.

A path $\gamma$ is a sequence $\left(x_{0}, \ldots, x_{k}\right)$ of consecutive incident or coinciding elements of $\Delta$. An elementary homotopy is a deletion or insertion of an element $x$ between two elements $y$ and $z$, where $\{x, y, z\}$ is a flag. A trivial path is a path $(x)$ consisting of a single element, and this is by definition also elementary homotopic to the path $(x, x)$. Two paths are called homotopic if they can be transformed into each other by a series of elementary homotopies. Finally, the geometry $\Delta^{0}(C)$ is called simply connected if it is connected and if every closed path contained in it is homotopic to a trivial path (with all intermediate elementary homotopic paths contained in $\Delta^{0}(C)$ ). It is rather easy to see that simple connectivity only makes sense in polar spaces of rank at least 3 . If a geometry $\Gamma$ is connected but not simply connected, then it admits a universal cover, which is a simply connected geometry admitting a local isomorphism onto $\Gamma$ (a local isomorphism is an epimorphism inducing isomorphisms on the rank 2 residues). The size of any fiber is called the covering degree of $\Gamma$. Hence $\Gamma$ is simply connected if and only if it has covering degree 1.

Given a geometry $\Gamma$, let $\Sigma$ be its geometric realization as a simplicial complex. Then $\Gamma$ is simply connected if and only if $\Sigma$ is simply connected as a topological space (see e.g. [11. Chapter 12]). Similarly, $\Gamma$ is connected if and only if $\Sigma$ is path connected.

In [1], Peter Abramenko encountered the problem of determining for which thick polar spaces $\Delta$ of rank 3 the geometry $\Delta^{0}(C)$, with $C$ any chamber of $\Delta$, is simply connected. He proved [1, Proposition 11, p. 66] that this was the case for all polar spaces of rank 3 , except for $\mathrm{W}(5, q), q=2,3,4, \mathrm{Q}(6, q), q=3, \mathrm{Q}^{-}(7, q), q=2,3, \mathrm{H}\left(5, q^{2}\right), q=2$, and except possibly for $\mathrm{W}(5, q), q=5,7,8,9,11, \mathrm{Q}(6, q), q=5,7,9,11,13, \mathrm{Q}^{-}(7, q)$, $q=4,5,7,8,9,11, \mathrm{H}\left(5, q^{2}\right), q=3,4,5, \mathrm{H}\left(6, q^{2}\right), q=2,3$, and all non-embeddable thick polar spaces.

In this paper, we settle all of these open cases and prove the following theorems.

Theorem 2.1. Let $\Delta$ be a thick polar space of rank 3, and let $C$ be a chamber of $\Delta$. Then $\Delta^{0}(C)$ is simply connected if $\Delta$ is isomorphic to a non-embeddable polar space.

Theorem 2.2. Let $\Delta$ be a thick polar space of rank 3, and let $C$ be a chamber of $\Delta$. Then $\Delta^{0}(C)$ is simply connected if $\Delta$ is isomorphic to $\mathrm{W}(5, q), q=5,7,8,9,11, \mathrm{Q}(6, q)$, $q=5,7,9,11,13, \mathrm{Q}^{-}(7, q), q=4,5,7,8,9,11, \mathrm{H}\left(5, q^{2}\right), q=3,4,5, \mathrm{H}\left(6, q^{2}\right), q=2,3$. 
Moreover, if $\Delta \cong \mathrm{W}(5,4)$ then the covering degree is 4 , and if $\Delta \cong \mathrm{Q}(6,3)$ then the covering degree is 81 .

For some more information on the covers of $\mathrm{W}(5,4)$ and $\Delta \cong \mathrm{Q}(6,3)$, see Proposition 5.2 . Combined with [1, Proposition 11, p. 66], we can now state the following.

Corollary 2.3. Let $\Delta$ be a thick polar space of rank 3, and let $C$ be a chamber of $\Delta$. Then $\Delta^{0}(C)$ is simply connected, except for the following cases:

(1) $\Delta \cong \mathrm{W}(5,2), \mathrm{W}(5,3), \mathrm{Q}^{-}(7,2), \mathrm{H}\left(5,2^{2}\right)$, where the covering degree is $\infty$;

(2) $\Delta \cong \mathrm{W}(5,4)$, where the covering degree is 4 ;

(3) $\Delta \cong \mathrm{Q}(6,3)$, where the covering degree is 81 .

The paper is organized as follows. In the next section, we introduce the necessary notions to handle the non-embeddable polar spaces, in particular we recall the recent construction of these polar spaces by De Bruyn \& Van Maldeghem [4. In Section 4 , we prove Theorem 2.1 for non-embeddable polar spaces. In Section 5, we report on the small cases to complete the proofs of Theorem 2.2 and Corollary 2.3 .

\section{Preliminaries on non-embeddable polar spaces}

Let $\mathbb{K}$ be a commutative field, and let $\mathbb{O}$ be a Cayley-Dickson division algebra with center $\mathbb{K}$. The Moufang projective plane over $\mathbb{O}$ is the projective plane $P G(2, \mathbb{O})$ with point set $\{(\infty),(m),(x, y): m, x, y \in \mathbb{O}\}$ and line set $\{[\infty],[x],[m, k]: x, m, k \in \mathbb{O}\}$, and with incidence relation $*$ defined by $(x, y) *[x] *(\infty) *[\infty] *(m) *[m, k]$ and

$$
(x, y) *[m, k] \text { if and only if } y=m x+k .
$$

According to Tits [13], there exists a unique polar space $\Delta$ of rank 3 with planes isomorphic to the Moufang projective plane over $\mathbb{O}$. We give the coordinate description which can be found in [4].

Let $\infty$ be a symbol not belonging to $\mathbb{O}$. Then the point set of $\Delta$ is the set $\left\{(\infty),\left(x_{1}\right),\left(x_{1}, x_{2}\right),\left(x_{1}, x_{2} ; k\right),\left(x_{1}, x_{2}, x_{3} ; k\right),\left(x_{1}, x_{2}, x_{3}, x_{4} ; k\right): x_{1}, x_{2}, x_{3}, x_{4} \in \mathbb{O}, k \in \mathbb{K}\right\}$. The point $(\infty)$ is called the point of type 0 . If $x_{1}, x_{2}, x_{3}, x_{4} \in \mathbb{O}$ and $k \in \mathbb{K}$, then $\left(x_{1}\right)$ is called a point of type $1,\left(x_{1}, x_{2}\right)$ is called a point of type $2,\left(x_{1}, x_{2} ; k\right)$ is called a point of type $3,\left(x_{1}, x_{2}, x_{3} ; k\right)$ is called a point of type 4 and $\left(x_{1}, x_{2}, x_{3}, x_{4} ; k\right)$ is called a point of type 5 . The planes of $\Delta$ are the following subsets of points, subdivided into eight types, where in each case $a, b, s \in \mathbb{O}$.

Type I. The plane $[\infty]$ consists of the following points:

$$
\begin{aligned}
p_{[\infty], 1}(a, b) & :=(a, b), \\
p_{[\infty], 2}(s) & :=(s), \\
p_{[\infty], 3} & :=(\infty) .
\end{aligned}
$$


Type II. For every $k \in \mathbb{K}$, the plane $[k]$ consists of the following points:

$$
\begin{aligned}
p_{[k], 1}(a, b) & :=(a, b ; k), \\
p_{[k], 2}(s) & :=(s), \\
p_{[k], 3} & :=(\infty) .
\end{aligned}
$$

Type III. For every $x \in \mathbb{O}$ and every $k \in \mathbb{K}$, the plane $[x ; k]$ consists of the following points:

$$
\begin{aligned}
p_{[x ; k], 1}(a, b) & :=(x, a, b ; k), \\
p_{[x ; k], 2}(s) & :=\left(-x^{\sigma}, s\right), \\
p_{[x ; k], 3} & :=(\infty) .
\end{aligned}
$$

Type IV. For every $x \in \mathbb{O}$ and all $k, l \in \mathbb{K}$, the plane $\alpha:=[x ; k, l]$ consists of the following points:

$$
\begin{aligned}
p_{\alpha, 1}(a, b) & :=\left(a, x+l a, b ; k+x^{\sigma} a+a^{\sigma} x+l a^{\sigma+1}\right), \\
p_{\alpha, 2}(s) & :=\left(x^{\sigma}, s ; l\right), \\
p_{\alpha, 3} & :=(\infty) .
\end{aligned}
$$

Type V. For all $x_{1}, x_{2} \in \mathbb{O}$ and every $k \in \mathbb{K}$, the plane $\alpha:=\left[x_{1}, x_{2} ; k\right]$ consists of the following points:

$$
\begin{aligned}
p_{\alpha, 1}(a, b) & :=\left(-x_{2}^{\sigma},-x_{1}^{\sigma}, a, b ; k\right), \\
p_{\alpha, 2}(s) & :=\left(s, x_{1}+x_{2} s\right), \\
p_{\alpha, 3} & :=\left(x_{2}\right) .
\end{aligned}
$$

Type VI. For all $x_{1}, x_{2} \in \mathbb{O}$ and all $k, l \in \mathbb{K}$, the plane $\alpha:=\left[x_{1}, x_{2} ; k, l\right]$ consists of the following points:

$$
\begin{aligned}
p_{\alpha, 1}(a, b) & :=\left(-x_{2}^{\sigma}, a, x_{1}^{\sigma}+k a, b ; l+x_{1} a+a^{\sigma} x_{1}^{\sigma}+k a^{\sigma+1}\right), \\
p_{\alpha, 2}(s) & :=\left(s, x_{1}+x_{2} s ; k\right), \\
p_{\alpha, 3} & :=\left(x_{2}\right) .
\end{aligned}
$$

Type VII. For all $x_{1}, x_{2}, x_{3} \in \mathbb{O}$ and all $k, l \in \mathbb{K}$, the plane $\alpha:=\left[x_{1}, x_{2}, x_{3} ; k, l\right]$ consists of the following points:

$$
\begin{aligned}
p_{\alpha, 1}(a, b) & :=\left(a,-x_{3}^{\sigma}+x_{1} a, b, x_{2}^{\sigma}+k a-x_{1}^{\sigma} b ; l+x_{2} a+a^{\sigma} x_{2}^{\sigma}+k a^{\sigma+1}\right), \\
p_{\alpha, 2}(s) & :=\left(x_{1}, s, x_{2}+x_{3} s ; k\right), \\
p_{\alpha, 3} & :=\left(-x_{1}^{\sigma}, x_{3}\right) .
\end{aligned}
$$


Type VIII. For all $x_{1}, x_{2}, x_{3} \in \mathbb{O}$ and all $k, l, m \in \mathbb{K}$, the plane $\alpha:=\left[x_{1}, x_{2}, x_{3} ; k, l, m\right]$ consists of the following points:

$$
\begin{aligned}
p_{\alpha, 1}(a, b):= & \left(a, b, x_{3}{ }^{\sigma}+l b+x_{1} a, x_{2}{ }^{\sigma}+k a+x_{1}^{\sigma} b ;\right. \\
& \left.m+x_{2} a+a^{\sigma} x_{2}^{\sigma}+x_{3} b+b^{\sigma} x_{3}{ }^{\sigma}+k a^{\sigma+1}+l b^{\sigma+1}+\left(a^{\sigma} x_{1}^{\sigma}\right) b+b^{\sigma}\left(x_{1} a\right)\right), \\
p_{\alpha, 2}(s):= & \left(s, x_{1}+l s, x_{2}+x_{3} s ; k+x_{1}^{\sigma} s+s^{\sigma} x_{1}+l s^{\sigma+1}\right), \\
p_{\alpha, 3}:= & \left(x_{1}^{\sigma}, x_{3} ; l\right) .
\end{aligned}
$$

Each of the above point sets admits a natural bijection $\beta_{\alpha}$ onto the point set of the plane $\mathrm{PG}(2, \mathbb{O})$ by mapping the point $p_{\alpha, 1}(a, b)$ to the point $(a, b)$, the point $p_{\alpha, 2}(s)$ to the point $(s)$, and then point $p_{\alpha, 3}$ to $(\infty)$. Now, these planes determine the collinearity relation in $\Delta$, and collinearity determines the lines. It is proved in [4] that one can explicitly describe all lines of $\Delta$ as the inverse images of the point sets of the lines in PG $(2, \mathbb{O})$ under the bijections $\beta_{\alpha}$. Moreover, the collection of all planes above is precisely the family of all maximal singular subspaces of $\Delta$.

In particular, one can check that the intersection of two planes is either empty, a single point, or a line of $\Delta$. In [4, all lines are explicitly given independently of the planes as sets of points, and the lines are subdivided into twelve types. Here, we will only need two of those types. The lines of type $L$ are the lines of the planes $\alpha$ of type VIII not containing $p_{\alpha, 3}$, and the lines of type $K$ are the lines of the planes $\alpha$ of type VIII containing $p_{\alpha, 3}$ but not $p_{\alpha, 2}(0)$. Note that each line of type L also occurs in a (unique) plane of type VII, and each line of type $\mathrm{K}$ occurs in a (unique) plane of type VI. Two points will be called L-collinear if they are contained in a common line of type L; similarly for K-collinearity.

We define the chamber $C_{\infty}$ as the chamber consisting of the point $(\infty)$, the line through the points $(\infty)$ and $(0)$, and the unique plane $[\infty]$ of type I.

If two points $p$ and $q$ are collinear, then we write $p \perp q$, and if two singular subspaces $V$ and $W$ are contained in a common singular subspace, then we denote by $\langle V, W\rangle$ the unique singular subspace of smallest dimension containing both $V$ and $W$.

\section{Proof of Theorem 2.1}

Let $\Delta$ be the non-embeddable thick polar space of rank 3 related to the Cayley-Dickson division algebra $\mathbb{O}$ with center the commutative field $\mathbb{K}$. We keep the notation of the previous paragraph and we want to show that $\Delta^{0}\left(C_{\infty}\right)$ is simply connected. We follow the general strategy of Abramenko [1], namely, we define a filtration of $\Delta^{0}\left(C_{\infty}\right)$ (i.e., a sequence of nested subgeometries whose union is $\left.\Delta^{0}\left(C_{\infty}\right)\right)$ that begins with a "large" contractible subgeometry and preserves simple connectivity at each step.

Note that it follows from the fact that the point $(\infty)$ belongs to all planes of type I, II, III and IV that a point is opposite $(\infty)$ if and only if it has type 5 . Likewise, it is easy to see that a plane is disjoint from the plane [ $\infty$ ] (and hence opposite) if and only if it has 
type VIII. Finally, it also follows that the lines opposite $\langle(\infty),(0)\rangle$ are precisely those of type L. Hence $\Delta^{0}\left(C_{\infty}\right)$ is the geometry induced by the set

$$
Y:=\{\text { points of type } 5\} \cup\{\text { lines of type } \mathrm{L}\} \cup\{\text { planes of type } \mathrm{VIII}\} \text {. }
$$

Before defining the filtration of $Y$ we record a result to be used later.

Proposition 4.1. Suppose that $\Gamma$ is a thick generalized quadrangle in which every point and every line is incident with at least $n^{2}+n+2$ elements, $n \in \mathbb{N}, n \geq 1$. If $Z$ is a nonempty set of points and lines of $\Gamma$ such that for any element $z$ of $Z$ at most $n$ elements incident with $z$ in $\Gamma$ do not belong to $Z$, then the geometry induced by $Z$ is connected.

Proof. Let $x, y$ be two elements of $Z$. Without loss, we may assume $d(x, y)=3$. Indeed, if $d(x, y)=2$, then consider any element of $Z$ incident with $y$ not incident with $x$ (since there is a unique element in $\Gamma$ incident with both $x$ and $y$, and $n \geq 1$, such element certainly exists). If $d(x, y)=4$, then any element of $Z$ incident with $y$ is at distance 3 from $x$.

Since $n^{2}+n+2-n \geq n+2$, we can select $n+1$ elements $x_{0}, x_{1}, \ldots, x_{n}$ of $Z$ incident with $x$ and at distance 4 from $y$. Since for each $x_{i}, i=0,1, \ldots, n$, there are at most $n$ elements incident with $x_{i}$ and not belonging to $Z$, and since for each such element there is a unique element at distance 2 and incident with $y$, there are at least $\left(n^{2}+n+1\right)-n(n+1) \geq 1$ elements $y_{0}$ incident with $y$ such that the unique elements $z_{i}$ at distance 2 from $y_{0}$ incident with $x_{i}, i=0,1, \ldots, n$, belong to $Z$. If $u_{i}$ denotes the unique element incident with both $z_{i}$ and $y_{0}, i=0,1, \ldots, n$, then, by assumption, at least one of this, say $u_{0}$, belongs to $Z$, and so the path $\left(x, x_{0}, z_{0}, u_{0}, y_{0}, y\right)$ belongs to $Z$.

For our purposes, we will mostly need the previous result in the following setting.

Corollary 4.2. Let $\Gamma$ be a generalized quadrangle such that every element is incident with infinitely many other elements. Let $n \geq 1$ be an arbitrary natural number, and let $a_{1}, a_{2}, \ldots, a_{n}$ be $n$ elements of $\Gamma$. Then $\cap_{i=1}^{n} \Gamma^{0}\left(a_{i}\right)$ is connected.

Proof. Assume that $x \in \cap_{i=1}^{n} \Gamma^{0}\left(a_{i}\right)$. Let $i \in\{1,2, \ldots, n\}$. If $x$ is opposite $a_{i}$, then $\Gamma_{x}$ is contained in $\Gamma^{0}\left(a_{i}\right)$. If $d\left(x, a_{i}\right)=3$, then the only element of $\Gamma_{x}$ not contained in $\Gamma^{0}\left(a_{i}\right)$ is the unique one at distance 2 from $a_{i}$. If $d\left(x, a_{i}\right) \leq 2$, then obviously $x \notin \Gamma^{0}\left(a_{i}\right)$, a contradiction. Hence at most $n$ elements incident with $x$ are not contained in $\cap_{i=1}^{n} \Gamma^{0}\left(a_{i}\right)$.

So the corollary will follow from Proposition 4.1 if we show that $\cap_{i=1}^{n} \Gamma^{0}\left(a_{i}\right)$ is nonempty. Let $\ell$ be maximal with the property that there exists $x \in \cap_{i=1}^{\ell} \Gamma^{0}\left(a_{i}\right)$. Then $\ell \geq 1$. Suppose for a contradiction that $\ell<n$. Then $d\left(x, a_{\ell+1}\right) \leq 2$. By the previous paragraph applied to $\cap_{i=1}^{\ell} \Gamma^{0}\left(a_{i}\right)$, we know that there exists $y \in \cap_{i=1}^{\ell} \Gamma^{0}\left(a_{i}\right)$ with $y$ incident with $x$ and $d\left(y, a_{\ell+1}\right)=d\left(x, a_{\ell+1}\right)+1$. Hence, after at most three steps, we can find an element in $\cap_{i=1}^{\ell+1} \Gamma^{0}\left(a_{i}\right)$, contradicting the definition of $\ell$.

Recall that for an element $x$ of $\Delta$ we denote by $\Delta_{x}$ the set (or induced subgraph on the set) of neighbors of $x$ in $\Delta$. If $x$ is a point, then $\Delta_{x}$ is itself a generalized quadrangle; 
if $x$ is a line, then it is a generalized digon (a complete bipartite graph); if $x$ is a plane, then it is a projective plane.

Proposition 4.3. Let $q$ be a point of type 5. Then it is contained in a unique plane $\alpha_{q}$ of type $V$ and in a unique line $L_{q}$ intersecting $\langle(\infty),(0)\rangle$ nontrivially. Moreover, in the generalized quadrangle $\Delta_{q}$, the chambers $C$ opposite the chamber $C_{q}:=\left\{L_{q}, \alpha_{q}\right\}$ are precisely those for which $C \cup\{q\}$ is opposite $C_{\infty}$, i.e., $\Delta_{q}^{0}\left(C_{q}\right)=\Delta^{0}\left(C_{\infty}\right) \cap \Delta_{q}$.

Proof. The first assertions follow from the fact that $q$ is opposite $(\infty)$. The last assertion follows directly from Theorem 3.28 and Proposition 3.29 of [13].

We will define the filtration of $Y$ step-by-step. We will have $Y_{0} \subseteq Y_{1} \subseteq \cdots \subseteq Y_{9} \subseteq Y_{10}=$ $Y$, and we start by defining $Y_{0}$.

Throughout, put $p:=(0,0,0,0 ; 0) \in Y$ and let $Y_{0} \subseteq Y$ comprise the following subspaces:

(1) points of type 5 L-collinear with $p$;

(2) lines of type L containing a point of type 5 L-collinear with $p$ and whose point of type 5 with first coordinate 0 is either

(a) non collinear with $p$, or

(b) K-collinear with $p$;

(3) planes of type VIII containing a line as in (2)(b) above.

To understand the structure of $Y_{0}$, note that no point of type 5 with first coordinate 0 is L-collinear with $p$, and points of type 5 with first coordinate 0 that are K-collinear with $\mathrm{p}$ are contained in a plane of type VIII as in (3). If a line $M$ of type L contains a point of type $5 \mathrm{~L}$-collinear with $p$, and if its point of type 5 with first coordinate 0 is also collinear with $p$, but not K-collinear, then the plane spanned by $p$ and $M$ is not of type VIII, and hence we cannot directly contract that line inside $Y_{0}$. This motivates the conditions in (2).

Proposition 4.4. The geometry $Y_{0}$ can be contracted onto $p$.

Proof. Indeed, we first contract every plane of type VIII containing a line as in (2)(b) onto that line. Then we contract every line of $Y_{0}$ as in (2)(a) onto its unique point of type 5 collinear with $p$, and every line of type $\mathrm{L}$ as in $(2)(\mathrm{b})$ onto the plane it spans together with $p$. Every point of type 5 L-collinear with $p$ is contracted onto the line it spans together with $p$, and every plane in $Y_{0}$ is contracted onto $p$. Finally, every line in $Y_{0}$ containing $p$ is contracted onto $p$.

The previous arguments can be summarized as: for any subspace $U$ in $Y_{0}$, the subspace defined by the points in $U$ collinear with $p$ also belongs to $Y_{0}$ (and we will denote that subspace by $U^{\perp p}$ ), and for any $U$ in $Y_{0}$ all of whose points are collinear with $p$, the subspace $\langle p, U\rangle$ also belongs to $Y_{0}$. 
Concerning the points of $Y_{0}$ it is appropriate to note that every point of $Y_{0} \backslash\{p\}$ has nonzero first coordinate. Conversely, one easily checks that every point in $\Delta$ of type 5 with nonzero first coordinate and collinear with $p$, is L-collinear with $p$. This makes it rather easy to recognize points of $Y_{0}$.

The planes in $Y_{0}$ admit a simple characterization via coordinates.

Proposition 4.5. Let $\alpha=\left[x_{1}, x_{2}, x_{3} ; k, l, m\right]$. Then $\alpha \in Y_{0}$ if and only if either $(i)$ $x_{2}=x_{3}=m=0$ or $(i i) x_{3} \neq 0$ and $m \neq 0$. Moreover, $p \in \alpha$ in case $(i)$ but not in case (ii).

Proof. We have $p_{\alpha, 1}(0,0)=\left(0,0, x_{3}^{\sigma}, x_{2}^{\sigma} ; m\right)$, so $p \in \alpha$ (and hence $\alpha \in Y_{0}$ ) if and only if $x_{2}=x_{3}=m=0$. If $p \notin \alpha$, then $\alpha^{\perp p}$ is a line, and hence $\alpha \in Y_{0}$ if and only if $\alpha^{\perp p} \in Y_{0}$. But $\alpha^{\perp p}$ has type $\mathrm{L}$ if and only if $p_{\alpha, 3}$ is not collinear with $p$ (by definition of type L), and this happens if and only if $x_{3} \neq 0$ (indeed, if $x_{3}=0$, then the plane $\left[x_{1}, 0,0 ; 0, l, 0\right]$ contains both $p$ and $p_{\alpha, 3}$; if $x_{3} \neq 0$, then any plane $\beta$ of type VIII through $p_{\alpha, 3}$ has third coordinate nonzero, implying that the point $p_{\beta, 1}(0,0)$ has nonzero third coordinate). Noting that a point of type 5 with first coordinate 0 and collinear with $p$, is $\mathrm{K}$-collinear with $p$ if and only if its second coordinate is distinct from 0 , we see that, if $x_{3} \neq 0$, the point of type 5 with first coordinate 0 in $\alpha^{\perp p}$ is K-collinear with $p$ if and only if $p_{\alpha, 1}(0,0)$ is not collinear with $p$, and this happens if and only if $m \neq 0$ (indeed, the points of type 5 with first and second coordinate 0 contained in a common plane of type V, VI or VII all have a common last coordinate, as is easily checked; if $m=0$, then $\left(0,0, x_{3}^{\sigma}, x_{2}^{\sigma} ; 0\right), x_{3} \neq 0$, and $(0,0,0,0 ; 0)$ are contained in the plane $\left.[0,0 ; 0]\right)$.

Now define $Y_{1}$ as follows (where $\mathbb{K}^{\times}=\mathbb{K} \backslash\{0\}$; we will also use $\mathbb{O}^{\times}=\mathbb{O} \backslash\{0\}$ ):

$$
Y_{1}=Y_{0} \cup \bigcup_{m \in \mathbb{K}^{\times}}\{q \in Y: q \text { is a point K-collinear with and distinct from }(0,0,0,0 ; m)\} \text {. }
$$

The points $(0,0,0,0 ; m)$ have special properties.

Lemma 4.6. For all $m \in \mathbb{K}$ we have $(\infty)^{\perp} \cap(0,0,0,0 ; m)^{\perp}=(\infty)^{\perp} \cap p^{\perp}$. In particular, no point of type 5 collinear with $(0,0,0,0 ; 0)$ is collinear with $(0,0,0,0 ; m), m \in \mathbb{O}^{\times}$.

Proof. One easily calculates that

$$
(\infty)^{\perp} \cap(0,0,0,0 ; m)^{\perp}=\left\{(0),\left(x_{1}, 0\right),\left(x_{1}, 0 ; k\right),\left(x_{1}, x_{2}, 0 ; k\right): x_{1}, x_{2} \in \mathbb{O}, k \in \mathbb{K}\right\},
$$

which is independent of $m$.

Proposition 4.7. For any $q \in Y_{1} \backslash Y_{0}$, the subgeometry $Y_{0} \cap \Delta_{q}$ is connected.

Proof. Let $m \in \mathbb{K}^{\times}$. An arbitrary plane $\alpha$ of type VIII containing the point $p_{\alpha, 1}(0,0)=$ $(0,0,0,0 ; m)$ has coordinates $\left[x_{1}, 0,0 ; k, l, m\right], x_{1} \in \mathbb{O}, k, l \in \mathbb{K}$. An arbitrary point in that plane K-collinear with and distinct from $(0,0,0,0 ; m)$ is just an arbitrary point in $\alpha$ with first coordinate equal to 0 and second distinct from 0 , i.e., $q=\left(0, b, l b, x_{1}^{\sigma} b ; m+l b^{\sigma+1}\right)$, $b \neq 0$. Lemma 4.6 implies that $q \notin Y_{0}$. 
By Lemma 4.6, a point of type unequal to 5 is collinear with $p$ if and only if it is collinear with $(0,0,0,0 ; m)$. Hence lines in $\Delta_{q} \cap Y$ opposite $\langle q,(0,0,0,0 ; m)\rangle$ are exactly those whose point of type 4 is not collinear with $p$. As $p$ is not collinear with $q$, such a line's unique point $r$ collinear with $p$ has type 5. Moreover, the first coordinate of $r$ is nonzero as $r$ and $q$ are L-collinear but not K-collinear. It follows that $p$ and $r$ are L-collinear. Hence the lines of $Y_{0} \cap \Delta_{q}$ are precisely the lines of $\Delta_{q}\left(C_{q}\right) \cap \Delta_{q}(\{\langle q,(0,0,0,0 ; m)\rangle, \beta\})$, where $\beta$ is any plane through $\langle q,(0,0,0,0 ; m)\rangle$.

It is straightforward to calculate that, if a plane $\beta=\left[x_{1}^{\prime}, x_{2}^{\prime}, x_{3}^{\prime} ; k^{\prime}, l^{\prime}, m^{\prime}\right]$ contains the point $q$, then $x_{3}^{\prime}=\left(l-l^{\prime}\right) b^{\sigma}$ and $m^{\prime}=m-\left(l-l^{\prime}\right) b^{\sigma+1}$. By Proposition 4.5, such a plane belongs to $Y_{0}$ if and only $x_{3}^{\prime} \neq 0$ and $m^{\prime} \neq 0$.

Since $b \neq 0$, the equality $x_{3}^{\prime}=0$ is equivalent with $l=l^{\prime}$. This is equivalent with saying that the unique point $r$ of type 3 of $\beta$ can be written as $\left(x_{1}^{\prime \sigma}, 0 ; l\right)$. A plane $\gamma=\left[x_{1}^{\prime \prime}, x_{2}^{\prime \prime} ; k^{\prime \prime}, l^{\prime \prime}\right]$ of type VI contains $q$ if and only if $x_{1}^{\prime \prime}=\left(l-k^{\prime \prime}\right) b^{\sigma}, x_{2}^{\prime \prime}=0$ and $l^{\prime \prime}=m+\left(k^{\prime \prime}-l\right) b^{\sigma+1}$. Such a plane contains $r$ if and only if $k^{\prime \prime}=l$; indeed, this is necessary as the last coordinate of $r$, which is $l$, must be equal to the third coordinate of $\gamma$, which is $k^{\prime \prime}$, but it is also sufficient since $k^{\prime \prime}=l$ implies $x_{1}=0$. Hence, the unique plane of type VI through $q$ and $r$ has coordinates $[0,0 ; l, m]$, and it follows that a plane $\left[x_{1}^{\prime}, x_{2}^{\prime}, x_{3}^{\prime} ; k^{\prime}, l^{\prime}, m^{\prime}\right], x_{1}^{\prime}, x_{2}^{\prime}, x_{3}^{\prime} \in \mathbb{O}, k^{\prime}, l^{\prime}, m^{\prime} \in \mathbb{K}$, containing $q$ satisfies $x_{3}^{\prime} \neq 0$ if and only if it intersects the plane $[0,0 ; l, m]$ only in $q$, hence if and only if those two planes define opposite elements in the generalized quadrangle $\Delta_{q}$.

Likewise, with similar calculations, one shows that the condition $m^{\prime} \neq 0$ translates into $\left[x_{1}^{\prime}, x_{2}^{\prime}, x_{3}^{\prime} ; k^{\prime}, l^{\prime}, m^{\prime}\right]$ being opposite $\gamma^{\prime}=\left[m b^{-1}, 0 ; l-m b^{-\sigma-1}, 0\right]$ in $\Delta_{q}$. It is now easy to see that

$$
Y_{0} \cap \Delta_{q}=\Delta_{q}^{0}\left(C_{q}\right) \cap \Delta_{q}^{0}(\{\langle q,(0,0,0,0 ; m)\rangle, \gamma\}) \cap \Delta_{q}^{0}\left(\left\{\langle q,(0,0,0,0 ; m)\rangle, \gamma^{\prime}\right\}\right) .
$$

The result now follows from Corollary 4.2 .

Proposition 4.8. $Y_{1}$ is simply connected.

Proof. By Proposition 4.4, the geometry $Y_{0}$ is contractible, hence simply-connected. Let $q \in Y_{1} \backslash Y_{0}$. The geometry induced on $\{q\} \cup\left(\Delta_{q} \cap T_{0}\right.$ is contractable onto $q$, hence it is simply connected. Also, the intersection $Y_{0} \cap \Delta_{q}$ is connected as a geometry; hence its geometric realization is a path-connected topological space. Using the Seifert-Van Kampen theorem for topological spaces, we now see that the geometric realization of the geometry induced on $Y_{0} \cup\{q\}$ is simply connected. We can repeat this argument for a finite number of elements $q=q_{0}, q_{1}, \ldots, q_{\ell}$ of $Y_{1} \backslash Y_{0}$ and obtain that the geometry induced on $Y_{0} \cup\left\{q_{0}, q_{1}, \ldots, q_{\ell}\right\}$ us simply connected. Since a closed path in $Y_{0}$ contains only a finite number of elements of $Y_{1} \backslash Y_{0}$, this implies that evey closed path is homotopic equivalent to the trivial path. Hence $Y_{1}$ is a simply connected geometry.

We now add some lines to $Y_{1}$ and define

$$
Y_{2}=Y_{1} \cup\left\{L: L \text { is a line of type } \mathrm{L} \text { containing a point of } Y_{1} \backslash Y_{0}\right\} \text {. }
$$


In order to show that $\Delta_{L} \cap Y_{1}$ is connected for $L \in Y_{2} \backslash Y_{1}$, we only need to show that $L$ is contained in some plane of $Y_{0}$, as $\Delta_{L}$ is complete bipartite.

Proposition 4.9. Each line $L \in Y_{2} \backslash Y_{1}$ is contained in a plane of $Y_{0}$, and hence $Y_{2}$ is simply connected.

Proof. Let the line $L$ contain the point $q=\left(0, b, l b, d ; m+l b^{\sigma+1}\right)$, with $b \in \mathbb{O}^{\times}, d \in \mathbb{O}$ and $l, m \in \mathbb{K}$. Let the unique point of type 4 on $L$ have coordinates $\left(w_{1}, w_{2}, w_{3} ; i\right) \in \mathbb{O}^{3} \times \mathbb{K}$. As in the previous proof, one calculates that a plane $\beta=\left[x_{1}^{\prime}, x_{2}^{\prime}, x_{3}^{\prime} ; k^{\prime}, l^{\prime}, m^{\prime}\right]$ contains $q$ if and only if

$$
\left\{\begin{array}{l}
x_{2}^{\prime}=d-x_{1}^{\prime \sigma} b^{\sigma}, \\
x_{3}^{\prime}=\left(l-l^{\prime}\right) b^{\sigma}, \\
m^{\prime}=m-\left(l-l^{\prime}\right) b^{\sigma+1} .
\end{array}\right.
$$

If we choose $l^{\prime} \notin\left\{l, l-m b^{-\sigma-1}\right\}$, which is always possible as $|\mathbb{K}|>2$, then $\beta$ contains $L$ and belongs to $Y_{0}$.

An argument similar as that in the proof of Proposition 4.8 shows that $Y_{2}$ is simply connected.

We now add some planes to $Y_{2}$ and define

$$
\begin{aligned}
Y_{3}=Y_{2} & \cup\left\{\left[x_{1}, x_{2}, x_{3} ; k, l, 0\right]: x_{3} \in \mathbb{O}^{\times}, x_{1}, x_{2} \in \mathbb{O}, k, l \in \mathbb{K}\right\} \\
\cup & \left.\cup\left[x_{1}, x_{2}, 0 ; k, l, m\right]: x_{1} \in \mathbb{O}, x_{2} \in \mathbb{O}^{\times}, k, l \in \mathbb{K}, m \in \mathbb{K}^{\times}\right\} .
\end{aligned}
$$

Proposition 4.10. Let $\alpha \in Y_{3} \backslash Y_{2}$. Then $\Delta_{\alpha} \cap Y_{2}$ is connected, and hence $Y_{3}$ is simply connected.

Proof. Let $A$ be the set of points in $\Delta_{\alpha} \cap Y_{0}$, let $B$ be the set of points in $\Delta_{\alpha} \cap\left(Y_{1} \backslash Y_{0}\right)$, and let $W$ be the set of lines in $\Delta_{\alpha} \cap Y_{2}$. Note that all points of $A$ have a nonzero first coordinate (indeed, every such point is L-collineair with $p$ and distinct from $p$ since either $x_{2} \neq 0$ or $\left.x_{3} \neq 0\right)$ and all points in $B$ have as first coordinate 0 . So $A \cap B=\emptyset$. We first show that $A \neq \emptyset \neq B$.

- $\alpha=\left[x_{1}, x_{2}, x_{3} ; k, l, 0\right]$, with $x_{3} \in \mathbb{O}^{\times}, x_{1}, x_{2} \in \mathbb{O}, k, l \in \mathbb{K}$. The point of type 3 in $\alpha$ is $\left(x_{1}^{\sigma}, x_{3} ; l\right)$, which is never collinear with $p$, since $x_{3} \neq 0$. Hence $\alpha^{\perp p}$ is a line of type $\mathrm{L}$, and so $A \neq \emptyset$ in this case. Also, $p_{\alpha, 1}\left(0, x_{3}^{\sigma}\right)$ is $\mathrm{K}$-collinear with $\left(0,0,0,0 ; x_{3}^{\sigma}\right)$ since both points have 0 as first coordinate and lie in the common plane $\left[x_{3}^{-1} x_{2}+x_{1}, 0,0 ; 0,1+l, x_{3}^{\sigma+1}\right]$. So $B \neq \emptyset$.

- $\alpha=\left[x_{1}, x_{2}, 0 ; k, l, m\right]$, with $x_{1} \in \mathbb{O}, x_{2} \in \mathbb{O}^{\times}, k, l \in \mathbb{K}, m \in \mathbb{K}^{\times}$. The point of type 3 in $\alpha$ is $\left(x_{1}^{\sigma}, 0 ; l\right)$, which is always collinear with $p$, and we also have that neither $p_{\alpha, 1}(0,0)=\left(0,0,0, x_{2}^{\sigma} ; m\right)$ nor $p_{\alpha, 2}(0)=\left(0, x_{1}, x_{2} ; k\right)$ is collinear with $p$ (all this because $x_{2} \neq 0$ and a plane of type VII or VIII containing $p$ must have second coordinate 0$)$. Hence every point in $\alpha$ distinct from $p_{\alpha, 3}$ collinear with $p$ has type 5 and has nonzero first coordinate, hence belongs to $Y_{0}$ and to $A$. Also, the point $p_{\alpha, 1}(0, b)=\left(0, b, l b, x_{2}^{\sigma}+x_{1}^{\sigma} b ; m+l b^{\sigma+1}\right)$ is K-collinear with and distinct from $(0,0,0,0 ; m)$, for every $b \in \mathbb{O}^{\times}$, see the first paragraph of the proof of Proposition 4.7. Hence $B \neq \emptyset$. 
Now, if a line $L$ in $\alpha$ belongs to $Y_{0}$, then it contains a point of $A$. Likewise, if a line $L$ in $\alpha$ belongs to $Y_{2} \backslash Y_{0}$, then it contains a point of $B$. Moreover, every line of type $\mathrm{L}$ through any point of $B$ is contained in $Y_{2}$. Noting that all lines connecting a point of $A$ with a point of $B$ have type $\mathrm{L}$, we immediately deduce that $A \cap B \cap W$ is connected.

Note that a plane $\left[x_{1}, x_{2}, x_{3} ; k, l, m\right], x_{1}, x_{2}, x_{3} \in \mathbb{O}, k, l, m \in \mathbb{K}$, belongs to $Y \backslash Y_{3}$ if and only if $x_{3}=0$ and either $x_{2}=0$ and $m \neq 0$, or $x_{2} \neq 0$ and $m=0$. These will be added in two distinct steps $\left(Y_{5}\right.$ and $Y_{7}$, respectively).

Now we again add some points.

$$
Y_{4}=Y_{0} \cup \bigcup_{m \in \mathbb{K}^{\times}}\{q \in Y: q \text { is a point L-collinear with and distinct from }(0,0,0,0 ; m)\} \text {. }
$$

We first establish the general form of a point in $Y_{4} \backslash Y_{1}$. Firstly, an arbitrary plane $\alpha$ of type VIII containing the point $p_{\alpha, 1}(0,0)=(0,0,0,0 ; m)$ has coordinates $\left[x_{1}, 0,0 ; k, l, m\right]$, $x_{1} \in \mathbb{O}, k, l \in \mathbb{K}$. An arbitrary point in that plane L-collinear with and distinct from $(0,0,0,0 ; m)$ is just an arbitrary point in $\alpha$ with first coordinate, say $a \in \mathbb{O}$, unequal 0 , i.e.,

$$
q=\left(a, b, l b+x_{1} a, k a+x_{1}^{\sigma} b ; m+k a^{\sigma+1}+l b^{\sigma+1}+\left(a^{\sigma} x_{1}^{\sigma}\right) b+b^{\sigma}\left(x_{1} a\right)\right), a \neq 0 .
$$

Proposition 4.11. Let $q \in Y_{4} \backslash Y_{3}$. Then $\Delta_{q} \cap Y_{3}$ is connected and hence $Y_{4}$ is simply connected.

Proof. Put $q=\left(a, b, l b+x_{1} a, k a+x_{1}^{\sigma} b ; m+k a^{\sigma+1}+l b^{\sigma+1}+\left(a^{\sigma} x_{1}^{\sigma}\right) b+b^{\sigma}\left(x_{1} a\right)\right)$, as above and put $r=\left(0,0,0, m a^{-\sigma} ; 0\right)$. It is clear that every plane of type VIII incident with $r$ must have the third and last coordinate equal to 0 . Let $\beta=\left[x_{1}^{\prime}, x_{2}^{\prime}, 0 ; k^{\prime}, l^{\prime}, 0\right]$, with $x_{1}^{\prime}, x_{2}^{\prime} \in \mathbb{O}$ and $k^{\prime}, l^{\prime} \in \mathbb{K}$ arbitrary, and suppose $q \in \beta$. The description of planes of type VIII implies

$$
\left\{\begin{aligned}
l b+x_{1} a & =l^{\prime} b+x_{1}^{\prime} a, \\
k a+x_{1}^{\sigma} b & =x_{2}^{\prime \sigma}+k^{\prime} a+x_{1}^{\prime \sigma} b, \\
m+k a^{\sigma+1}+l b^{\sigma+1}+\left(a^{\sigma} x_{1}^{\sigma}\right) b+b^{\sigma}\left(x_{1} a\right) & =x_{2}^{\prime} a+a^{\sigma} x_{2}^{\prime \sigma}+k^{\prime} a^{\sigma+1}+l^{\prime} b^{\sigma+1}+\left(a^{\sigma} x_{1}^{\prime \sigma}\right) b+b^{\sigma}\left(x_{1}^{\prime} a\right) .
\end{aligned}\right.
$$

Putting $L=l-l^{\prime}, K=k-k^{\prime}$ and $X_{1}=x_{1}-x_{1}^{\prime}$, we can rewrite this as

$$
\left\{\begin{aligned}
L b+X_{1} a & =0 \\
K a+X_{1}^{\sigma} b & =x_{2}^{\prime \sigma} \\
m+K a^{\sigma+1}+L b^{\sigma+1}+\left(a^{\sigma} X_{1}^{\sigma}\right) b+b^{\sigma}\left(X_{1} a\right) & =x_{2}^{\prime} a+a^{\sigma}{x_{2}^{\prime}}^{\sigma} .
\end{aligned}\right.
$$

Remembering $K, L \in \mathbb{K}$, we deduce from the first equation that $X_{1}$ belongs to the skew field generated by $a$ and $b$; then the second equation says that also $x_{2}^{\prime}$ belongs to this skew field. Hence all elements in this system of equations belong to a common skew field, and so we may remove the parentheses. It is now an elementary exercise to eliminate $L$ and $K$ from the first two equations, and then also $X_{1}$ disappears in the last equation, giving $m=x_{2}^{\prime} a$. Noting that $\left(0,0,0, x_{2}^{\prime \sigma} ; 0\right)$ always belongs to $\left[x_{1}^{\prime}, x_{2}^{\prime}, 0 ; k^{\prime}, l^{\prime}, 0\right]$, we conclude 
that $r \in \beta$. Hence a plane of type VIII containing $q$ has the form $\left[x_{1}^{\prime}, x_{2}^{\prime}, 0 ; k^{\prime}, l^{\prime}, 0\right]$, $x_{1}^{\prime}, x_{2}^{\prime} \in \mathbb{O}, k^{\prime}, l^{\prime} \in \mathbb{K}$, if and only if it contains $r$.

Now put $s=(0,0,0,0 ; m)$. It is clear that every plane of type VIII incident with $s$ must have second and third coordinate equal to 0 . Conversely, let $\gamma$ be an arbitrary plane with coordinates $\left[x_{1}^{\prime \prime}, 0,0 ; k^{\prime \prime}, l^{\prime \prime}, m^{\prime \prime}\right]$ and suppose $q \in \gamma$. Note that $\left(0,0,0,0 ; m^{\prime \prime}\right) \in \gamma$. But then Lemma 4.6 implies $m=m^{\prime \prime}$, yielding $s \in \gamma$. Hence a plane of type VIII containing $q$ has the form $\left[x_{1}^{\prime \prime}, 0,0 ; k^{\prime \prime}, l^{\prime \prime}, m^{\prime \prime}\right], x_{1}^{\prime \prime} \in \mathbb{O}, k^{\prime \prime}, l^{\prime \prime}, m^{\prime \prime} \in \mathbb{K}$, if and only if it contains $s$. We conclude that the planes of $Y_{3}$ in $\Delta_{q}$ are precisely the planes of $\Delta_{q}^{0}\left(C_{q}\right) \cap \Delta_{q}^{0}(\langle r, q\rangle) \cap \Delta_{q}^{0}(\langle s, q\rangle)$.

Noting that $p$ is not collinear with $q$, a line $R$ of type 5 through $q$ is not contained in $Y_{0}$ if and only if its unique point of type 4 is collinear with $p$, or its unique point of type 5 with first coordinate 0 is collinear with $p$. Now, by Lemma 4.6, the point of type 4 on $R$ is collinear with $p$ if and only if it is collinear with $s$ if and only if $\langle q, s\rangle$ is collinear with $R$ in $\Delta_{q}$. Now let $u$ be a point of type 5 with first coordinate 0 . Suppose first that $u$ is collinear with $p$. Since $u$ is not L-collinear with $p$, one verifies (see [4]) that the line $\langle u, p\rangle$ is contained in a unique plane of type either $\mathrm{V}$ or $\mathrm{VI}$, of the form $[0,0 ; 0]$ or $[0,0 ; k, 0], k \in \mathbb{K}$ respectively. But such a plane automatically contains $r$. Conversely, if $u$ is collinear with $r$, then the same argument implies that $p$ is collinear with $u$. Hence we have shown that the lines of $Y_{0}$ in $\Delta_{q}$ are precisely those of $\Delta_{q}^{0}\left(C_{q}\right) \cap \Delta_{q}^{0}(\langle r, q\rangle) \cap \Delta_{q}^{0}(\langle s, q\rangle)$. By Corollary 4.2, this is connected. Proposition 4.9 implies that $\Delta_{q} \cap Y_{3}$ is connected.

We now add half of the missing planes to $Y_{4}$ and define

$$
Y_{5}=Y_{4} \cup\left\{\left[x_{1}, 0,0 ; k, l, m\right]: x_{1} \in \mathbb{O}, k, l \in \mathbb{K}, m \in \mathbb{K}^{\times}\right\} .
$$

Proposition 4.12. Let $\alpha \in Y_{5} \backslash Y_{4}$. Then $\Delta_{\alpha} \cap Y_{4}$ is connected and hence $Y_{5}$ is simply connected.

Proof. Let $\alpha=\left[x_{1}, 0,0 ; k, l, m\right], x_{1} \in \mathbb{O}, k, l \in \mathbb{K}, m \in \mathbb{K}^{\times}$. We note that $(0,0,0,0 ; m) \in$ $\alpha$, hence by the definition of $Y_{1}$ and $Y_{4}$, all other points of type 5 of $\alpha$ are contained in $Y_{4}$. By the definition of $Y_{2}$, all lines in $\alpha$ of type $\mathrm{L}$ containing a point $p_{\alpha, 1}(0, b), b \in \mathbb{O}^{\times}$, belong to $Y_{4}$. This implies easily that $\Delta_{\alpha} \cap Y_{4}$ is connected (in fact, it suffices to find two lines through each point of $\alpha$ that belongs to $Y_{4}$, but here we found infinitely many).

So far, $Y_{5}$ contains all lines of type $\mathrm{L}$ all of whose points are collinear with $p$. It also contains all lines of type $\mathrm{L}$ with a unique point $q$ collinear with $p$ such that $q$ has type 5 and is L-collinear with $p$, or such that $q$ has type 4 and the line contains a point Kcollinear with some $(0,0,0,0 ; m), m \in \mathbb{K}^{\times}$. Since all lines of type $\mathrm{L}$ contain some point collinear with $(0,0,0,0 ; m)$, for all $m \in \mathbb{O}$, it is natural to first concentrate on special cases of that collinearity.

Also, there are still lines in $Y$ not incident with any point of $Y_{5}$. We cannot add those lines, as such a line $M$ would give rise to non-connected $\Delta_{M} \cap Y_{5}$. For instance, one checks that the line $T$ of the plane $\alpha=[0,1,1 ; 0,0,0]$ containing the points $p_{\alpha, 2}(-1)$ and $p_{\alpha, 1}(0, x)$, with $x \in \mathbb{O} \backslash \mathbb{K}$, does not contain any point of type 5 collinear with any point $(0,0,0,0 ; m), m \in \mathbb{K}$. 
Hence we now add some specific lines to $Y_{5}$ and define $Y_{6}$ as follows.

$$
Y_{6}=Y_{5} \cup\left\{\left\langle q, q^{\prime}\right\rangle: q=(a, b, c, d ; k) \in Y_{4}, b \neq 0 \text { and } q \perp q^{\prime}=\left(a^{\prime}, 0, c^{\prime}, d^{\prime} ; k^{\prime}\right), a^{\prime} \notin\{0, a\}\right\} .
$$

By definition, every line $R \in Y_{6} \backslash Y_{5}$ contains at least one point of $Y_{4}$; so to prove connectivity of $\Delta_{R} \cap Y_{5}$, it suffices to prove that there is at least one plane of $Y_{5}$ incident with $R$.

Proposition 4.13. There is at least one plane of $Y_{3}$ containing any line $R$ of $Y_{6} \backslash Y_{5}$. Consequently, $\Delta_{R} \cap Y_{5}$ is connected and hence $Y_{6}$ is simply connected.

Proof. Every line of type L is incident with infinitely many planes of type VIII, and exactly one of type VII. Suppose two planes $\alpha_{1}, \alpha_{2}$ of type VIII meet in $R$ and let $\alpha_{i}=\left[x_{i}, y_{i}, 0 ; k_{i}, l_{i}, 0\right], x_{i}, y_{i} \in \mathbb{O}, y_{i} \neq 0, k_{i}, l_{i} \in \mathbb{K}, i=1,2$.

Let $R=\left\langle q, q^{\prime}\right\rangle$ with $q=(a, b, c, d ; k) \in Y_{4}, b \neq 0, q \perp q^{\prime}=\left(a^{\prime}, 0, c^{\prime}, d^{\prime} ; k^{\prime}\right), a^{\prime} \notin\{0, a\}$. Let $r=\left(w_{1}, w_{2}, w_{3} ; i\right)$ be the unique point of type 4 in $R$. Note that this implies, by the definition of lines in $\Delta$, that $b-w_{1} a=0-w_{1} a^{\prime}$, hence $w_{1}=b\left(a-a^{\prime}\right)^{-1} \neq 0$. Since $r \in \alpha_{i}, i=1,2$, we easily deduce

$$
\left\{\begin{array}{c}
x_{1}-x_{2}=-\left(l_{1}-l_{2}\right) w_{1} \\
k_{1}-k_{2}=\left(l_{1}-l_{2}\right) w_{1}^{\sigma+1}
\end{array}\right.
$$

Since $a^{\prime} \neq 0$ and $w_{1} \neq 0, R$ contains a point $p_{\alpha_{i}, 1}(0, e)$, with $e \neq 0, i=1,2$. This easily yields, by comparing the third coordinate of $p_{\alpha_{1}, 1}(0, e)=p_{\alpha_{2}, 1}(0, e), l_{1} b=l_{2} b$, hence $l_{1}=l_{2}$, and by the above equalities, $x_{1}=x_{2}$ and $k_{1}=k_{2}$. From the fourth coordinate of $p_{\alpha_{1}, 1}(0, e)=p_{\alpha_{2}, 1}(0, e)$ follows $y_{1}=y_{2}$, hence $\alpha_{1}=\alpha_{2}$. It follows that there is at most one plane of type VIII not in $Y_{5}$ that contains $R$. The proposition is proved.

Now we add all remaining planes to $Y_{6}$.

$$
Y_{7}=Y_{6} \cup\left\{\left[x_{1}, x_{2}, 0 ; k, l, 0\right]: x_{1} \in \mathbb{O}, k, l \in \mathbb{K}, x_{2} \in \mathbb{O}^{\times}\right\} .
$$

Proposition 4.14. For any plane $\alpha \in Y_{7} \backslash Y_{6}$, we have that $\Delta_{\alpha} \cap Y_{6}$ is connected and hence $Y_{7}$ is simply connected.

Proof. Put $\alpha=\left[x_{1}, x_{2}, 0 ; k, l, 0\right]: x_{1} \in \mathbb{O}, k, l \in \mathbb{K}, x_{2} \in \mathbb{O}^{\times}$. Let us determine the points of $Y_{6}$ in $\alpha$. Note first that $p_{\alpha, 3}=\left(x_{1}^{\sigma}, 0 ; l\right)=p_{\beta, 3}$, with $\beta=\left[x_{1}, 0,0 ; 0, l, 0\right] \ni p$. Also, $p_{\alpha, 1}(0,0)=\left(0,0,0, x_{2}^{\sigma} ; 0\right)=p_{\gamma, 1}\left(0, x_{2}^{\sigma}\right) \ni p$, where $\gamma=[0,0 ; 0]$. Hence the line $\alpha^{\perp p}$ has type $\mathrm{K}$ and all of its points of type 5 have 0 as first coordinate and so are not L-collinear with $p$. Consequently, there are no points of $Y_{0}$ in $\alpha$.

By Lemma 4.6, every point $r_{m}:=(0,0,0,0 ; m), m \in \mathbb{K}$, is collinear with $p_{\alpha, 3}$. Hence all points of type 5 collinear with $r_{m}$ have the same first coordinate (distinct from 0 by Lemma 4.6 again), and so there is one with second coordinate 0 , say $p_{\alpha, 1}(a, 0)$. Since $a \neq 0, p_{\alpha, 1}(a, 0)$ cannot be K-collinear with $r_{m}$. So, no point of $\alpha$ belongs to $Y_{1}$. The calculation in the first part of the proof of Proposition 4.11 shows that $p_{\alpha, 1}(a, 0)$ is Lcollinear to $r_{m}$ if and only if $m=x_{2} a$, hence $a$ ranges over all $\mathbb{K}$-multiples of $x_{2}^{-1}$. Since 
every line of type $\mathrm{L}$ in $\alpha$ contains a point of type 5 with first coordinate $x_{2}^{-1}$, it suffices to show that two arbitrary points of $Y_{4}$ in $\alpha$ can be joined by a path of $Y_{6}$ in $\alpha$. By the definition of lines in $Y_{6} \backslash Y_{5}$, every point of $Y_{4}$ in $\alpha$ is connected by a single line to $p_{\alpha, 1}\left(x_{2}^{-1}, 0\right)$, except for the points $p_{\alpha, 1}\left(x_{2}^{-1}, b\right), b \in \mathbb{O}^{\times}$, and the points $p_{\alpha, 1}(a, 0), a \neq x_{2}^{-1}$. Every point $p_{\alpha, 1}(a, 0), a \neq x_{2}^{-1}$ is connected with every point $p_{\alpha, 1}\left(x_{2}^{-1}, b\right), b \in \mathbb{O}^{\times}$, so we have at most two connected components. Now, picking two distinct $m, m^{\prime} \in \mathbb{K} \backslash\{0,1\}$, we see these two sets are connected since the point $p_{\alpha, 1}\left(m x_{2}^{-1}, 1\right)$ is connected with $p_{\alpha, 1}\left(m^{\prime} x_{2}^{-1}, 0\right)$.

We now add all the lines having a point in $Y_{7}$ (and we know that there are still others, see the example of the line $T$ above).

$$
Y_{8}=Y_{7} \cup\left\{M: M \text { is a line of type } \mathrm{L} \text { containing a point in } Y_{4}\right\} .
$$

Since every line in $Y_{8} \backslash Y_{7}$ is by definition incident with a point of $Y_{7}$, and since $Y_{7}$ contains all planes of type VIII, and every line of type $L$ is inside a plane of type VIII, we know that $\Delta_{M} \cap Y_{7}$ is connected, for $M \in Y_{8} \backslash Y_{7}$, and hence $Y_{8}$ is simply connected.

We now add all remaining points to $Y_{8}$ and define

$$
Y_{9}=Y_{8} \cup\{q: q \text { is a point of type } 5\} .
$$

Proposition 4.15. For any point $q \in Y_{9} \backslash Y_{8}$, we have that $\Delta_{q} \cap Y_{8}$ is connected and hence $Y_{9}$ is simply connected.

Proof. First note that $Y_{8}$ contains all planes of $Y$, hence if we consider a line $M \ni q$ of $Y_{8}$, then all but exactly one planes of $\Delta_{q}$ incident with $M$ belong to $\Delta_{q} \cap Y_{8}$. Now let $\alpha=\left[x_{1}, x_{2}, x_{3} ; k, l, m\right], x_{1}, x_{2}, x_{3} \in \mathbb{O}, k, l, m \in \mathbb{K}$, be a plane through $q$. We show that at most two lines of $\alpha$ through $q$ do not belong to $Y_{8}$. It will then follow from Proposition 4.1 that $\Delta_{q} \cap Y_{8}$ is connected.

Suppose first that $x_{3} \neq 0$. Then there is a unique point $r=p_{\alpha, 2}\left(-x_{3}^{-1} x_{2}\right)$ of type 4 with third coordinate 0 . Let $M$ be a line in $\alpha$ through $q$ distinct from (1) the line of type $\mathrm{K}$ through $q$, and (2) the line $\langle q, r\rangle$. Then $M$ has type $\mathrm{L}$ and the point $r_{M}$ on $M$ of type 4 has third coordinate distinct from 0 . It is easily seen that this implies that $r_{M}$ is not collinear with $p$, hence, by Lemma 4.6, $r_{M}$ is not collinear with any point $\left(0,0,0,0 ; m^{\prime}\right)$, for any $m^{\prime} \in \mathbb{K}$. Hence, again using Lemma 4.6, all points $s_{m^{\prime}}$ collinear with $\left(0,0,0,0 ; m^{\prime}\right), m^{\prime} \in \mathbb{K}$, and incident with $M$ are distinct, and so at least one has first coordinate nonzero, implying that it is L-collinear with the corresponding point $\left(0,0,0,0 ; m^{\prime}\right)$. This, in turn, implies that $M$ belongs to $Y_{8}$.

Hence we may assume that $x_{3}=0$. If $x_{2} \neq 0$, then no point of type 4 in $\alpha$ has third coordinate equal to 0 , and so the argument of the previous paragraph implies that only the line of type $\mathrm{K}$ through $q$ in $\alpha$ does not belong to $Y_{8}$, but all other lines in $\alpha$ through $q$ do.

Hence we may assume that $x_{2}=x_{3}=0$. In this case $(0,0,0,0 ; m) \in \alpha$, and then it follows from the definitions of $Y_{1}, Y_{2}, Y_{4}$ and $Y_{8}$ that all lines of type L through $q$ in $\alpha$ belong to $Y_{8}$. 
Now that we have all points and planes of $Y$ in $Y_{9}$, we can add all remaining lines and define $Y_{10}=Y$. Clearly, for any line $M \in Y_{10}$, the geometry $\Delta_{M} \cap Y$ is connected and, once again, the Seifert-Van Kampen theorem, as used in the proof of Proposition 4.8, implies that $Y$ is simply connected. This completes the proof of Theorem 2.1 for the nonembeddable thick polar spaces.

\section{The small cases}

In this section we deal with some of the smallest finite polar spaces of rank 3 . Note that Abramenko already proved the following in [1, Example 8, p. 65]: Suppose $\Delta$ is one of $\mathrm{W}(5,2), \mathrm{W}(5,3), \mathrm{Q}^{-}(7,2)$ or $\mathrm{H}\left(5,2^{2}\right)$, and $C$ is a chamber of $\Delta$. Then the covering degree of $\Delta^{0}(C)$ is $\infty$. Moreover, he points out that computer calculations (not described in detail) show that $\mathrm{W}(5,4)$ and $\mathrm{Q}(6,3)$ are not simply connected, although the covering degree remained unknown.

We deal with the remaining open finite cases with the help of computer calculations performed using the GAP [7] computer algebra system and various of its extension packages ${ }^{1}$ For this, we follow an approach already outlined by Abramenko, which is group theoretic and does not directly involve the geometry.

Throughout this section, let $\Delta$ be a thick finite polar space of rank 3 . Let $\left(H,\left(U_{\alpha}\right)_{\alpha \in \Phi}\right)$ be a corresponding root group datum, as defined in [3]. That is, $\Phi$ is a root system, the $U_{\alpha}$ are the root groups and $H$ normalizes the root groups. The group $G$, generated by $H$ and the root groups, acts naturally on $\Delta$. Modulo its center it is a subgroup of $\operatorname{Aut}(\Delta)$ (and often even equal).

Fix a base $\Pi=\left\{\alpha_{1}, \ldots, \alpha_{n}\right\}$ of $\Phi$ such that $\alpha_{1}, \alpha_{2}$ span a root system of type $B C_{2}$ and $\alpha_{2}, \alpha_{3}$ one of type $A_{2}$. This base yields a system of positive roots $\Phi_{+}$. Define $U_{i}:=U_{\alpha_{i}}$ for $1 \leq i \leq n$, and $U_{i j}:=\left\langle U_{i}, U_{j}\right\rangle$ for $1 \leq i<j \leq n$. Moreover let $U:=\left\langle U_{\alpha} \mid \alpha \in \Phi_{+}\right\rangle$, and let $C$ be the chamber of $\Delta$ stabilized by $U$. Then if $\Delta^{0}(C)$ is connected (which is always the case unless $\Delta \cong \mathrm{W}(5,2)$, see [1, Proposition 7 , p. 60]), the following holds:

$$
U=\left\langle U_{1}, U_{2}, U_{3}\right\rangle, \quad U_{1}=U_{12} \cap U_{13}, \quad U_{2}=U_{12} \cap U_{23}, \quad U_{3}=U_{13} \cap U_{23} .
$$

Denote by $\widetilde{U}$ the universal completion of the amalgam given on $U_{12}, U_{13}, U_{23}$ with respect to their intersections. Clearly the group $U$ is a completion of this amalgam, hence $U$ is a quotient of $\widetilde{U}$. More precisely, the following refinement of the Tits' lemma [14] is true (see also [1, Lemma 19, p. 64] and the discussion preceding it):

Lemma 5.1. There exists an exact sequence of the form

$$
1 \rightarrow \pi_{1}\left(\left|\Delta^{0}(C)\right|\right) \rightarrow \widetilde{U} \rightarrow U \rightarrow 1
$$

${ }^{1}$ The complete source code can be downloaded from the first author's homepage at http://www. quendi.de/math. 
The covering degree of $\Delta^{0}(C)$ equals the size of $\pi_{1}\left(\left|\Delta^{0}(C)\right|\right)$. Thus it is finite if and only if $\widetilde{U}$ is finite, and in that case equals $|\widetilde{U}| /|U|$. In the following we exploit exactly this relation. Note that we already know $|U|$ (its size is the product of the sizes of all root subgroups), so it remains to compute the size of $\widetilde{U}$, or prove that it is infinite.

First, we need to enter $\widetilde{U}$ into the computer. Since $\widetilde{U}$ is the amalgamated product of the groups $U_{12}, U_{13}, U_{23}$, we need the root groups $U_{1}, U_{2}, U_{3}$. To this end we construct a matrix representation for $U$ and the root groups, based on [3, Section 10], respectively the expositions of this material in [1, pp. 77-78 and 107-111] and [2, Example 7.136]. From this, it is straight-forward to first compute finite presentations for the groups $U_{i j}, 1 \leq i<j \leq 3$ and then for their amalgamated product $\widetilde{U}$. Note that [3] also describes presentations, which we could in principle also use. But by using the matrix representations, we can perform additional consistency checks on all obtained results (e.g. whether the matrices indeed preserve a suitable quadratic form).

There are in total five families of finite polar spaces we need to consider. In Table 1 we summarize some information about the groups occurring in each case.

\begin{tabular}{c|c|c||c||c|c||c|c|c}
$\Delta$ & $G$ & $|U|$ & $\left|U_{1}\right|$ & $\left|U_{2}\right|$ & $\left|U_{3}\right|$ & $\left|U_{12}\right|$ & $\left|U_{13}\right|$ & $\left|U_{23}\right|$ \\
\hline $\mathrm{W}(5, q)$ & $\mathrm{Sp}_{6}\left(\mathbb{F}_{q}\right)$ & $q^{9}$ & $q$ & $q$ & $q$ & $q^{4}$ & $q^{2}$ & $q^{3}$ \\
$\mathrm{Q}(6, q)$ & $\mathrm{O}_{7}\left(\mathbb{F}_{q}\right)$ & $q^{9}$ & $q$ & $q$ & $q$ & $q^{4}$ & $q^{2}$ & $q^{3}$ \\
$\mathrm{Q}^{-}(7, q)$ & ${ }^{2} \mathrm{O}_{8}\left(\mathbb{F}_{q}\right)$ & $q^{12}$ & $q^{2}$ & $q$ & $q$ & $q^{6}$ & $q^{3}$ & $q^{3}$ \\
$\mathrm{H}\left(5, q^{2}\right)$ & $\mathrm{U}_{6}\left(\mathbb{F}_{q}\right)$ & $q^{15}$ & $q$ & $q^{2}$ & $q^{2}$ & $q^{6}$ & $q^{3}$ & $q^{6}$ \\
$\mathrm{H}\left(6, q^{2}\right)$ & $\mathrm{U}_{7}\left(\mathbb{F}_{q}\right)$ & $q^{21}$ & $q^{3}$ & $q^{2}$ & $q^{2}$ & $q^{10}$ & $q^{6}$ & $q^{5}$
\end{tabular}

Table 1: Finite polar spaces of rank 3, groups of automorphisms and root group sizes.

The next step is to compute the size of $\widetilde{U}$. The usual approach to that is coset enumeration. However, doing this naïvely (by enumerating cosets of the trivial subgroup or some cyclic subgroups) is not feasible except in the very smallest cases, due to the quickly growing size of $\widetilde{U}$ and the resulting memory and time requirements. So a more refined approach is necessary. We employ the following three strategies:

1. To handle the cases where $\widetilde{U}$ is infinite, we compute the abelian invariants of the second derived subgroup $\widetilde{U}^{\prime \prime}$ of $\widetilde{U}$, that is, the quotient $\widetilde{U}^{\prime \prime} / \widetilde{U}^{\prime \prime \prime}$. We find the following, verifying the result from [1, Example 8, p. 65] that $\widetilde{U}$ is infinite in each of these cases:

(a) For $\Delta \cong \mathrm{W}(5,2)$, we have $\widetilde{U}^{\prime \prime} / \widetilde{U}^{\prime \prime \prime} \cong \mathbb{Z}^{2}$.

(b) For $\Delta \cong \mathrm{W}(5,3)$, we have $\widetilde{U}^{\prime \prime} / \widetilde{U}^{\prime \prime \prime} \cong \mathbb{Z}^{12}$.

(c) For $\Delta \cong \mathrm{Q}^{-}(7,2)$, we have $\widetilde{U}^{\prime \prime} / \widetilde{U}^{\prime \prime \prime} \cong \mathbb{Z}^{50}$.

(d) For $\Delta \cong \mathrm{H}\left(5,2^{2}\right)$, we have $\widetilde{U}^{\prime \prime} / \widetilde{U}^{\prime \prime \prime} \cong \mathbb{Z}^{12}$.

2. We may exploit that $U_{i j}$ injects into every completion of the amalgam. Since $|\widetilde{U}|=\left|U_{i j}\right| \cdot\left|\widetilde{U}: U_{i j}\right|$, and we know $\left|U_{i j}\right|$, it suffices to compute $\left|\widetilde{U}: U_{i j}\right|$, which can be done using coset enumeration. The larger the subgroup, the more efficiently 
this yields a result. Consulting Table 1 , we see that $U_{12}$ is the best choice. The expected index (if the covering degree is 1 ) then ranges between $q^{5}$ and $q^{11}$.

Using this strategy and the GAP package ACE [8], we computed the following: The group $U$ is isomorphic to $\widetilde{U}$ if $\Delta$ is isomorphic to $\mathrm{W}(5, q), 5 \leq q \leq 16$, or $\mathrm{Q}(6, q)$, $5 \leq q \leq 16$, or $\mathrm{Q}^{-}(7, q), 3 \leq q \leq 13$, or $\mathrm{H}\left(5, q^{2}\right), 3 \leq q \leq 5$, or $\mathrm{H}\left(6, q^{2}\right), 2 \leq q \leq 5$. For $\mathrm{W}(5,4)$ the covering degree is 4 , and for $\mathrm{Q}(6,3)$ the covering degree is 81 .

3. While the coset enumeration already covers all previously unknown cases, we now discuss a second, more effective approach to handle the cases with finite covering degree. This second approach is completely different and works for larger values of $q$ without running into memory limitations, which is the primary factor preventing the coset enumeration approach from being applied to more cases.

First, we use the GAP package NQ [10] to compute the maximal nilpotent quotient $U_{n q}$ of $\widetilde{U}$. Computing the size of $U_{n q}$ (or, for that matter, most other properties) is then simple and fast, as it is given by a so-called polycyclic presentation, which permits highly effective computations. The sole remaining obstacle is the verification that $U_{n q} \cong \widetilde{U}$. For this we apply a trick from [12, Section 11.8] involving rewriting systems: First a new presentation for $\widetilde{U}$ is constructed by adding new generators and relations that correspond to a polycyclic generating sequence in $U_{n q}$. Then the GAP package kbmag [9] is used to construct a confluent rewriting system from this. Finally, this confluent rewriting system can be used to compute the size of $\widetilde{U}$. But of course $\widetilde{U}$ and $U_{n q}$ are isomorphic if and only if they have the same size.

Using this approach, we computed the following: The group $U$ is isomorphic to $\widetilde{U}$ if $\Delta$ is isomorphic to $\mathrm{W}(5, q), 5 \leq q \leq 61$, or $\mathrm{Q}(6, q), 5 \leq q \leq 61$, or $\mathrm{Q}^{-}(7, q)$, $3 \leq q \leq 47$, or $\mathrm{H}\left(5, q^{2}\right), 3 \leq q \leq 11$, or $\mathrm{H}\left(6, q^{2}\right), 2 \leq q \leq 9$. For $\mathrm{W}(5,4)$ the covering degree is 4 , and for $\mathrm{Q}(6,3)$ the covering degree is 81 .

From the above discussion, Theorem 2.2 now follows.

We point out that for each of the five families of finite polar space of rank 3, our computations covered both previously known and unknown cases. Where the results were previously known, our computational results agree.

The above techniques also apply to the two cases with finite, non-trivial covering degree using GAP, where we computationally prove that $U_{n q} \cong \widetilde{U}$. Working with the group $U_{n q}$ then allows us to deduce the following.

Proposition 5.2. If $\Delta \cong \mathrm{W}(5,4)$, then $\widetilde{U}$ is a non-split central extension of $U$ over the module $\mathbb{F}_{2}^{2}$, and $H^{2}\left(U, \mathbb{F}_{2}^{2}\right)=\mathbb{F}_{2}^{50}$. The nilpotency classes of $U$ and $\widetilde{U}$ are equal to 4 each. If $\Delta \cong \mathrm{Q}(6,3)$, then $\widetilde{U}$ is a non-split non-central extension of $U$ over the module $\mathbb{F}_{3}^{4}$, and $H^{2}\left(U, \mathbb{F}_{3}^{4}\right)=\mathbb{F}_{3}^{13}$. The nilpotency classes of $U$ and $\widetilde{U}$ are equal to 5 and 9 , respectively.

Finally, we should mention that the same techniques can be applied to higher rank. In particular, we computed the following (note that for the cases over $\mathbb{F}_{2}$, the rank 3 subgeometries are not connected, which makes it necessary to consider all positive root 
subgroups, and not just those corresponding to a base of the root system).

Proposition 5.3. Let $\Delta$ be a thick polar space, let $C$ be a chamber of $\Delta$. Then $\Delta^{0}(C)$ is simply connected if $\Delta \cong W(7,3), W(7,4), \mathrm{Q}(8,3), \mathrm{Q}^{-}(9,2), \mathrm{H}\left(7,2^{2}\right)$, or if $\Delta \cong W(9,2)$. On the other hand, if $\Delta \cong W(7,2)$ then $\Delta^{0}(C)$ is not simply connected and the covering degree is $\infty$.

With some effort, and using a straight-forward generalization of [5], it should be possible to prove that the geometry induced by the set of chambers opposite a given chamber in any polar space of rank at least 4 is simply connected, with the sole exception of $W(7,2)$.

\section{References}

[1] P. Abramenko, Twin buildings and applications to S-arithmetic groups, Lect. Notes Math. 1641, Springer-Verlag, Berlin, 1996.

[2] P. Abramenko \& K. S. Brown, Buildings, Grad. Texts in Math. 248, Springer, New York, 2008.

[3] F. Bruhat \& J. Tits, Groupes réductifs sur un corps local, I., Inst. Hautes Etudes Sci. Publ. Math. 41 (1972), 5-251.

[4] B. De Bruyn \& H. Van Maldeghem, Non-embeddable polar spaces, Münster J. Math., to appear.

[5] A. Devillers \& B. Mühlherr, On the simple connectedness of certain subsets of buildings, Forum Math. 19 (2007), 955-970.

[6] A. Devillers, B. Mühlherr \& H. Van Maldeghem, Codistances in 3-spherical buildings, Math. Ann. 354 (2012), 297-329.

[7] The GAP Group, GAP - Groups, Algorithms, and Programming, Version 4.6.3; 2013, (http://www.gap-system.org).

[8] W. Gamble, A. Hukpke, G. Havas \& C. Ramsay, ACE - Advanced Coset Enumerator, A refereed GAP 4 package, see [7].

[9] D. Holt, kbmag - Knuth-Bendix on Monoids and Automatic Groups, A refereed GAP 4 package, see [7].

[10] W. Nickel, NQ - computing nilpotent factor groups of finitely presented groups, A refereed GAP 4 package, see [7].

[11] A. Pasini, Diagram Geometries, Clarendon Press, Oxford, 1994.

[12] C. Sims, Computation with finitely presented groups, Cambridge University Press, 1994.

[13] J. Tits, Buildings of spherical type and finite BN-pairs, Lect. Notes Math. 386, Springer-Verlag, Berlin, 1974. 
[14] J. Tits, Ensembles ordonnés, immeubles et sommes amalgamées, Bull. Soc. Math. Belg. Sér. A 38 (1986), 367-387.

[15] F. D. Veldkamp, Polar geometry I-V, Indag. Math. 21 (1959), 512-551, and Indag. Math. 22 (1959), 207-212. 
Answer to referee's comments.

We have corrected all linguistic errors pointed out by the referee, except the first one: the referee suggests to replace "more small" in "more small classical polar spaces" into "smaller", but the "more" refers to the spaces (so "more polar spaces which are small"). We have simply deleted "more".

There were two remarks concerning the content. The first one to comment on the structure of $Y_{0}$, and to expand the proof of its simply connectivity (contractibility). We have done this, see page 9 , the paragraph starting with "To understand the structure of $Y_{0} \ldots$ ". And we made a proposition of the contractibility of $Y_{0}$, see Proposition 4.4 on page 9 , with a 6-line proof now.

The second remark on the content was to state the Seifert-Van Kampen theorem for graphs. In fact, we use the ordinary Seifert Van Kampen theorem, but apply it to a geometric realisation of our simplical complexes. We give a more detailed account of this 